\title{
REA_03 - Immunoinformatics approach for epitope-based diagnosis of hemorraghic diseases caused by arenaviruses
}

Fernando de Paiva Conte ${ }^{1}$; Renata Carvalho de Oliveira ${ }^{2}$; Jorlan Fernandes de Jesus ${ }^{2 *}$; Elba Regina Sampaio de Lemos ${ }^{2}$; Rodrigo Nunes Rodrigues da Silva ${ }^{1}$.

${ }^{1}$ Fiocruz/Bio-Manguinhos;

${ }^{2}$ Fiocruz/IOC.

Introduction: Arenaviruses are rodent-borne pathogens that are important causes of hemorrhagic fever (HF) in Africa and South America. Human to human transmission plays a larger role in certain arenaviruses related outbreaks, for Lassa fever is estimated to cause up to 300,000 cases and 5,000 deaths per year in endemic regions of West Africa. HF cases have recently reemerged in Bolivia and Brazil with a high fatality rate, and several followups for close contacts. Although arenavirus infections have been limited to certain geographic areas, they pose as a serious challenge for local control of human cases and rodent reservoirs raise serious concerns about the potential for larger outbreaks in the future. Since arenaviruses are among the national compulsory notifiable diseases list and demand a biosafety level 4 for handling human samples, in this work we combined different in silico approaches to identify B-cell conserved regions on viral nucleoprotein of New World arenaviruses associated with hemorrhagic fever. Altogether, the results presented here could be used for new serologic diagnostic assays.

Objective: Identify B-cell linear immunogenic epitopes on viral nucleoprotein conserved among New World arenaviruses associated with hemorrhagic fever.

Methodology: In silico analysis were performed using the protein sequences of New World arenaviruses nucleoprotein (ANP) associated to hemorrhagic disease (Sabiá, Chapare, Machupo, Guanarito and Junín) obtained from NCBI. To predict immune antibody response targets, several protein features were assessed, including: surface exposure, flexibility, hydrophilicity, antigenicity and epitope location in protein quaternary structure. Linear Bcell epitopes were compared between: New World arenaviruses associated and nonassociated to hemorraghic diseases (Amapari, Aporé, Cupixi and Tacaribe) and Old World arenaviruses (Lassa and Lujo). Sabia NP ab initio homology modeling was carried out using Robbeta server. The best predicted models were chosen and the lowest energy one was selected. Resulting 3D structures were evaluated with Verify 3D and MolProbity.

Results: In silico analyses identified five potential B-cell linear and immunogenic targets on ANP (ANP1 to -5$)$. ANP-4 epitope shares a high degree of sequence conservation $(>90 \%)$ among all New World arenaviruses (associated or not) to hemorrhagic diseases and an intermediate similarity $(50<\mathrm{x}<65 \%)$ between Old World hemorrhagic arenaviruses. ANP-3 presents high degree of sequence conservation with Brazilian and Bolivian arenavirusesassociated to hemorrhagic diseases.

Conclusion: Immunoinformatics approach identified antibody targets that could be used for diagnosis of New World hemorrhagic arenaviruses.

Keywords: bioinformatics; epitope; arenavirus 